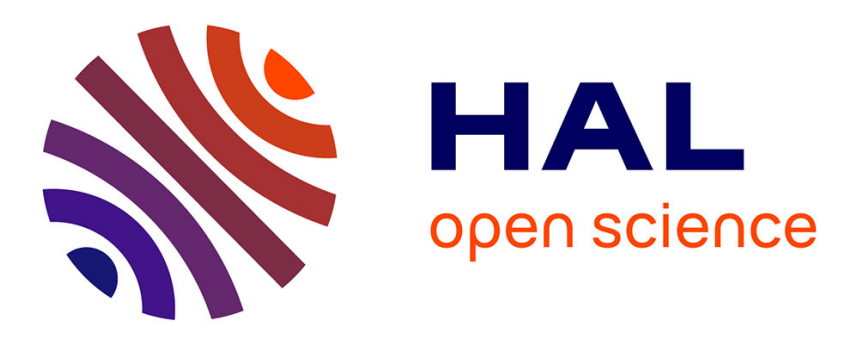

\title{
Bayesian network model with dynamic structure identification for real time diagnosis
}

\author{
Dang-Trinh Nguyen, Quoc Bao Duong, Éric Zamaï, Muhammad Kashif \\ Shahzad
}

\section{- To cite this version:}

Dang-Trinh Nguyen, Quoc Bao Duong, Éric Zamaï, Muhammad Kashif Shahzad. Bayesian network model with dynamic structure identification for real time diagnosis. 19th IEEE International Conference on Emerging Technologies and Factory Automation, Sep 2014, Barcelone, Spain. pp.8. hal-01058754

\section{HAL Id: hal-01058754 https://hal.science/hal-01058754}

Submitted on 28 Aug 2014

HAL is a multi-disciplinary open access archive for the deposit and dissemination of scientific research documents, whether they are published or not. The documents may come from teaching and research institutions in France or abroad, or from public or private research centers.
L'archive ouverte pluridisciplinaire HAL, est destinée au dépôt et à la diffusion de documents scientifiques de niveau recherche, publiés ou non, émanant des établissements d'enseignement et de recherche français ou étrangers, des laboratoires publics ou privés. 


\title{
Bayesian network model with dynamic structure identification for real time diagnosis
}

\author{
Dang Trinh Nguyen*, Quoc Bao Duong ${ }^{\dagger}$, Eric Zamai*, Muhammad Kashif Shahzad ${ }^{*}$ \\ ${ }^{*}$ Grenoble-INP, G-SCOP Laboratory \\ 46 avenue Felix Viallet, 38031 Grenoble Cedex, France \\ ${ }^{\dagger}$ A.I.P PRIMECA Dauphine Savoie \\ 740 Rue de la piscine BP 46, 38402 ST Martin D’Heres Cedex, France \\ \{Dang-Trinh.Nguyen, Quoc-Bao.Duong, Eric.Zamai, Muhammad-Kashif.Shahzad\}@ grenoble-inp.fr
}

\begin{abstract}
This paper proposes a method for real time diagnosis against product quality drifts in an automated manufacturing system. We use Logical Diagnosis model to reduce the search space of suspected equipment in the production flow, which is then formulated as a Bayesian network to compute risk priority for each equipment, using joint and conditional probabilities. The objective is to quickly and accurately localize the possible fault origins and support effective decisions on corrective maintenance. The key advantages offered by this method are (i) reduced unscheduled equipment breakdowns, and (ii) increased and stable production capacities, required for success in highly competitive and automated manufacturing systems. Moreover, this is a generic method and can be deployed on fully or semi automated manufacturing systems.
\end{abstract}

Index terms-Fault diagnosis, Automated Manufacturing Systems, Logical diagnosis, Bayesian network.

\section{INTRODUCTION}

Nowadays, manufacturing processes are more and more complex. One of main challenges for manufacturing domain is to improve and optimize the production process quality and equipment effectiveness during production operations. To improve product quality and reduce associated costs, fault isolation, detection and diagnosis techniques have been developed. For the fault diagnosis, many methods are proposed through a diagnosis model as presented in [1], [2] to localize more quickly and accurately the root causes of a detected failure. Theses model based methods are designed with flexibility to apply to different production systems.

However, the production system comprises of hundreds of equipment, monitored by thousands of sensors. Thus, we must analyze a large amount of production information for system control, monitoring and fault diagnosis. Generally, it is difficult to identify a diagnosis model and corresponding variables. Hence, probabilistic approaches are well suited techniques to analyze the large amount of production information and describe the behavior of system components as presented in [3]. These approaches can be performed without understanding the underlying structure of a production system [4]. Among the probabilistic approaches, the Bayesian network (BN) approach is widely used to identify a graphical structure model that describes relationships between variables in production system. So, its conditional probabilities will be calculated to provide the risk priorities and support corrective maintenance decisions. However, a difficulty of $\mathrm{BN}$ approaches is to identify the graphical structure during learning phase [5]. Due to the complexity of present-day manufacturing system, identification of this graphical structure is complex to be performed by a maintenance engineer [4]. Moreover, a production system depends on the products, recipes, equipment, maintenance and human factors. These elements are frequently changed due to the introduction of new production technologies and maintenance management philosophies. A change in one element may trigger effect to others. For example, to manufacture a new product, the corresponding technology, recipe, equipment, human factors may change. Consequently, we need to retrain the learning step of $\mathrm{BN}$ model to adapt with newly emerging situations of the production system. The time and workload for computation are very large, so that results in poor maintenance and additional costs.

Consequently, this paper focuses on potential diagnosis that use Logical Diagnosis model [6]. This reduces the search space for faulty equipment from a given production flow and optimizes the learning step for the subsequent BN. The BN model, based on the graphical structure, received from Logical Diagnosis model then computes joint and conditional probabilities for each node, to support corrective maintenance decisions. The proposed method enables real time diagnosis for corrective maintenance in fully or semi automated manufacturing systems.

The characteristics of case study and diagnosis objective are introduced in the next section. Section 3 presents a review on fault diagnosis methods. The Logical Diagnosis model that results in suspected equipment against a failure drift explained in Section 4, whereas Section 5 represents the principles of Bayesian network theory. The proposed diagnosis model is described in Section 6. We use an example to explain the application of proposed method as illustrated in Section 7. At the end of this paper, the conclusion and future perspectives are discussed in Section 8.

\section{CASE STUdy And Diagnosis ObJective}

\section{A. Case study}

Manufacturing processes evolve to adapt increasing demand diversity. Speed, reliability, flexibility, cost, rapid product innovation and quality are all related to design a manufacturing process [7]. Many types of manufacturing system presented in [8] support the design and management of manufacturing processes. In fact, the customers demands frequently change, and so, it leads to the changes in corresponding products. It 


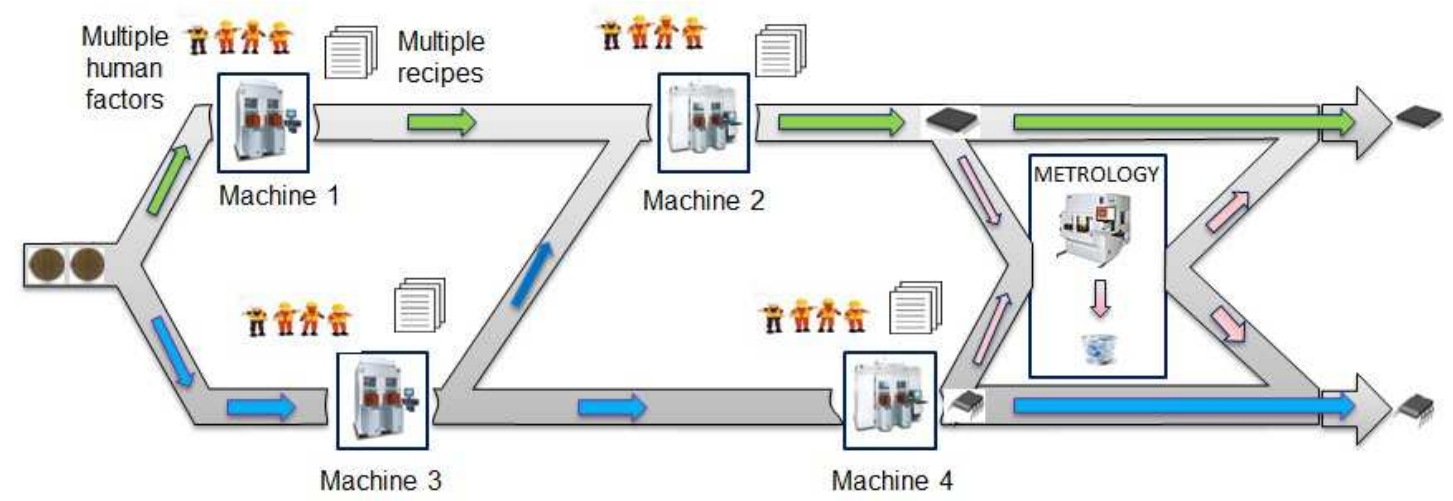

Fig. 1. A Flexible Manufacturing System

requires the flexibility of manufacturing system. This is reason to explain that flexible manufacturing systems (FMS) are widely used in complex and highly competitive manufacturing domains such as (micro-)electronic, automotive industry. FMS is a complex automated manufacturing system that consists of several production workshops, connected by an appropriate transport system. These production workshops and transport system are controlled by a control and automation system. The FMS is generally characterized by multiple products, production lines, recipes, and human factors. Generally, a complex system may have many production processes driven by a control law to perform demands of the control system. The structure of a production process describes product type, product lines and corresponding equipment in the operating part of controlled system. Any change in one of elements (such as product, equipment, recipe and human) through control law leads to adapt the existing processes.

Consequently, our research is based in the context of a general complex system under dynamic production environment with characteristics: multiple products, production lines, recipes, and human factors as shown in Fig.1.

\section{B. Diagnosis objective}

In complex engineering applications, systems can be composed of many components and subsystems, and the ways in which these elements interact will affect the way failures propagate within the subsystems and across subsystem boundaries [9]. For monitoring the execution of these elements, the hierarchical and modular controls are often used as presented in [10]. In this context, an automated manufacturing system is organised by Computer Integrated Manufacturing (CIM) architecture that contains : controlled system, product flow and control system, as shown in Fig.2. The controlled system consists of actuators and sensors. These sensors allow controlling and monitoring the executions of actuators and product flows. Each elementary part of the controlled system (actuators and sensors) is controlled by a local control module, and these two elements are called Functional Chain (FC) [11]. They receive and execute demands from the coordination level of control system. When a FC cannot correctly execute a demand, it implies that a fault is produced. A fault may be created by a component and can propagate to other components through production lines. Therefore, this failure propagation may have

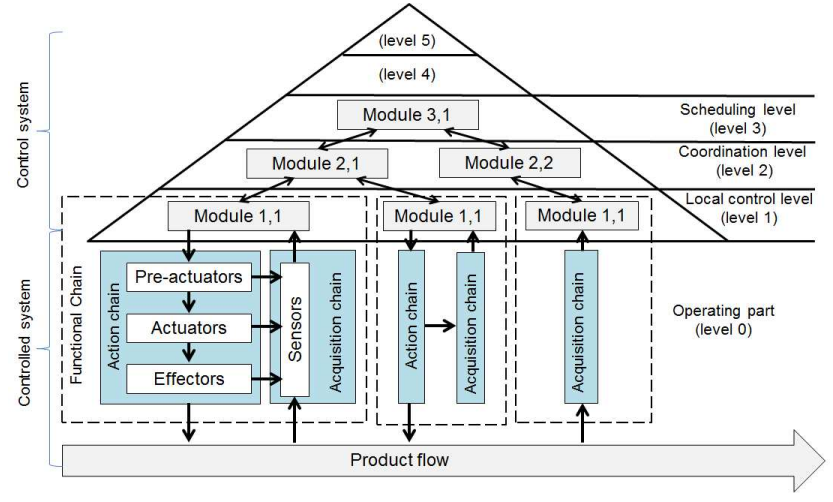

Fig. 2. CIM architecture of an automated production system

consequences on many components; and so, the new faults perhaps continue to occur. Thus, when a fault is detected by a metrology, its root causes may come from one or a part of elements of system. In summary, the diagnosis objective is to precisely and quickly locate the root causes to save recovery time (for return to a normal status) of the production system.

\section{FAULT DIAGNOSIS METHODS STATUS}

Diagnosis methods such as Fault Detection and Isolation (FDI) [12], Failure Mode and Effects Analysis (FMEA) [13] are classically used for fault diagnosis in a production equipment. An advantage of these approaches is its simple integration in the production system. From detection information and the symptoms of failure, these kinds of approaches are proposed to model the possible failures in the production system. However, it is not feasible to analyze a complex system to describe all the failures and their causes. When an unpredicted fault occurs, the diagnosis cannot provide a result without human supports. In a complex system, a fault can propagate from equipment to others through product flows and it may trigger the consequences to others system elements; and so, the unpredicted failures frequently occur. In this case, the existing failure model cannot explain such problem.

Other techniques based on failure propagation to analyze component dependencies are propagation graph [14] and temporal chronic [15]. These approaches are based on historical production data to locate components that are possible origins 
of a detected fault. However, there are many problems as follows: First, the actions of components in a system as presented in [14] are considered independent. In fact, several operations may use the same functional chain to execute the demands of control system. Thus, the corresponding actions are not independent. Second, these approaches do not analyse the behaviors of the functional chains (FC), so they cannot explain the consequences between elements of a FC and between different FCs. Finally, these approaches cannot reduce the size of the model. Due to the cyclical operation of the control system, a large amount of information from the production system provokes the problem of combinatory explosion. Consequently, we are especially interested in the Logical Diagnosis model proposed in [6]. In this model, a diagnosis function is proposed to characterize the historical information data of a controlled system to search the suspected potential fault origins in real time. Hence, this model provides a set of possible origins under the form of a directed graph, and its size is reduced by the exploitation of the controlled system observations. This is appropriate to diagnose the faults of production equipment and products. However, this model does not show suspect level of each member in this set of fault origins. It is difficult in deciding a maintenance order. In a complex system, the set of possible fault origins is still large and this approach would be extended to optimize corrective maintenance activities.

In order to evaluate the suspect level of potential failure candidates, the probabilistic approaches are widely used such as Neural network [16] and Bayesian network [3]. These approaches enable to calculate the probability values from the large database and associated variables in a production system. These probability values allow evaluating the suspect level (high or low) in order to support decision of a maintenance strategy. In particular, the BN models have the advantages that fit to be applied in manufacturing industry as explained in [3]. The methods based on $\mathrm{BN}$ are introduced in [4] to make a diagnosis in a multiple variables system. The Confidence level of feedback information is proposed in [17] to provide the probability value that shows the correct actions confidence of reported information from equipment. When the database is available, these approaches are practical tools for the corrective maintenance. However, they must be extended. The structure of confidence level model for information feedback, as presented in [17], is static with seven parameters. In the context of flexible manufacturing system with characteristics such as multiple products, production lines, recipes and human factors, the production situation often change as we presented in above section. Hence, the set of parameters that can have an impact on the equipment confidence is dynamic. In addition, the BN model must be updated with the information for new situations. In practice, when the information in database is available, learning approaches are often used for modelling BN as presented in [18]. In the learning approaches, a graphical structure and probabilistic rules are estimated from observed data. We can cite a number of learning approaches introduced in [5], [18] such as EM, Maximum likelihood, IC\&IC, K2, and Genetic algorithm. Many studies in [4], [18] show that these learning approaches are still complex in identifying variables as they depend strongly on expert opinions. The learning workload for computation is still large [18]. This spends too much time and is not appropriate for real time structural identification as it depends on the exploitation of databases. In fact, production environments are increasingly stressed by strong competition. It shows that the time for locating the root causes of failures and process recovery (return the process to a normal status) is very important. These challenges promote the researches to apply $\mathrm{BN}$ model for real time fault diagnosis and corrective maintenance optimization.

This paper proposes a diagnosis model that enables real time localization of the possible fault and root causes, thus dynamically computing conditional probabilities between a fault and its possible causes. This model is based on a Logical Diagnosis model and a BN model. The Logical Diagnosis model aides to reduce the workload of model identification for diagnosis model. It has four main steps: $(i)$ the Logical Diagnosis model provides a set of possible fault origins. The relationships of members in this set are used to construct a graphical structure, (ii) we use this structure for the BN model. This idea is to simplify the variables identification during learning phase in the $\mathrm{BN}$, (iii) the historical information of production system is used to estimate probabilistic rules in learning step of $\mathrm{BN}$ model, (iv) the conditional probabilities of nodes in structural model are computed. These probabilistic values allow evaluating the suspect level for each possible fault origin. In the next section, we will present the principles of the Logical Diagnosis model.

\section{Logical Diagnosis Model}

The Logical Diagnosis model proposed in [6] is one part in the treatment of failure propagation through a product in a complex production process. In this model, a diagnosis function is proposed to characterize the historical information from a controlled system in locating the possible origins of a detected fault. This diagnosis function is presented in three main points:

- First, a model generates in real time the normal operation of operating part and collects all the necessary information from coordination level for fault diagnosis through the Operation Models that are presented in [11].

\begin{tabular}{|c|c|c|}
\hline \multicolumn{3}{|c|}{ Operation $\mathrm{O}_{\mathrm{i}}$} \\
\hline \multicolumn{2}{|l|}{ Functional chain $\mathrm{ec}_{\mathrm{j}}$} & Service $\mathrm{Se}_{\mathrm{i}}$ \\
\hline \multicolumn{3}{|l|}{ During $\mathrm{Du}_{\mathrm{i}}$} \\
\hline \multicolumn{3}{|c|}{ Evolution of function chain $\mathrm{ec}_{\mathrm{i}}$} \\
\hline \multirow[t]{2}{*}{ Effect } & Pre-condition & Pre-contrain \\
\hline & $\operatorname{Pcd}\left(e c_{j}\right)$ & $\operatorname{PCt}\left(e_{j}\right)$ \\
\hline V. IC $\left(E f T\left(e c_{j}\right)\right)$ & Condition & Contrain \\
\hline Transient effect EfT $\left(e c_{j}\right)$ & $\mathrm{Cd}\left(\mathrm{ec}_{\mathrm{j}}\right)$ & $\mathrm{Ct}\left(\mathrm{ec}_{\mathrm{j}}\right)$ \\
\hline \multicolumn{3}{|l|}{ V IC $\left(\operatorname{EfF}\left(e_{\jmath}\right)\right)$} \\
\hline \multicolumn{3}{|l|}{ Final effect $E f F\left(e c_{j}\right)$} \\
\hline \multicolumn{3}{|c|}{ Premier Evolution of flow of product ea $a_{i, 1}$} \\
\hline \multirow[t]{2}{*}{ Effet } & Pre-condition & Pre-contrain \\
\hline & $\operatorname{Pcd}\left(e a_{i, 1}\right)$ & $\operatorname{PCt}\left(e a_{i, 1}\right)$ \\
\hline V IC $\left(E f T\left(e a_{i, 1}\right)\right)$ & Condition & Contrain \\
\hline Transient effect EfT $\left(e a_{i, 1}\right)$ & $\mathrm{Cd}\left(\mathrm{ea}_{\mathrm{i}, 1}\right)$ & $\mathrm{Ct}\left(\mathrm{ea}_{\mathrm{i}, 1}\right)$ \\
\hline \multicolumn{3}{|l|}{ V IC $\left(\operatorname{EfF}\left(e a_{i, 1}\right)\right)$} \\
\hline Final effect $\operatorname{EfF}\left(e a_{i, 1}\right)$ & & \\
\hline
\end{tabular}

Fig. 3. Operation Model 
An Operation Model contains information of Functional Chains (equipment, sensors and local control modular), (Pre-)conditions, (Pre-)constraints and effects of operations as shown in Fig.3. The model for diagnosis describes a graphical structure of a production process that consists of system components (as illustrated in Fig.4) and its relationships following product flows (the arrows $\rightarrow$ in Fig.4).

- Second, a mechanism to reduce this model is developed based on the exploitation of the controlled system observations. Following an operation in this model, if the information provided by these two elements is not coherent, the controlled system sends to the coordination level a faulty execution report. If the coordination level does not receive a faulty execution report from these Functional Chains, it can conclude that the corresponding element is reliable. These reliable elements (the black nodes in Fig.4) will be removed from the model, while the suspect elements (the white nodes in Fig.4) will be retained.

- Finally, a mechanism is defined, based on the failure propagation approach (the dash arrows $\rightarrow$ in Fig.4), that allows us searching the possible origins and the possible consequences of a fault when the model receives a faulty execution report from the Functional Chain as described in Fig.4.

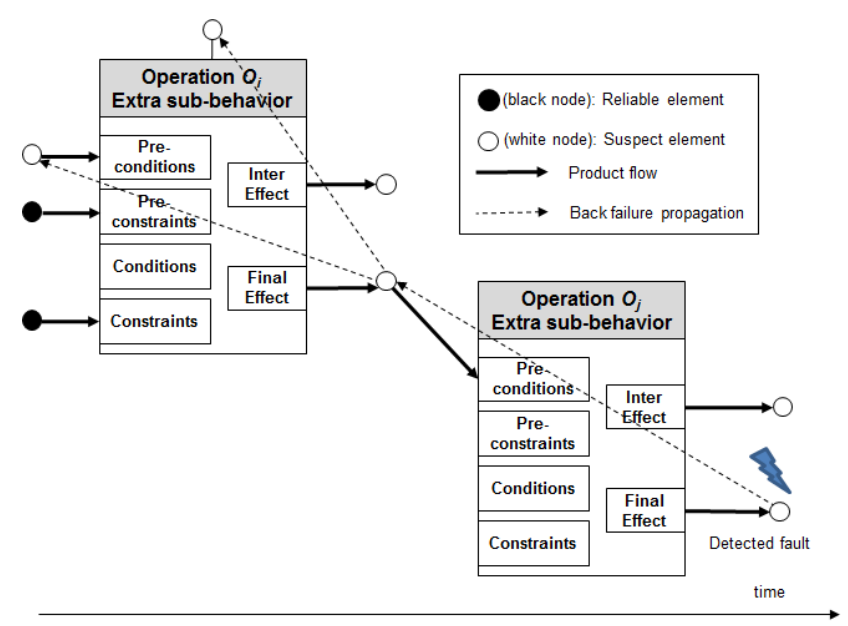

Fig. 4. Structural model

Indeed, this Logical Diagnosis model provides dynamically a structural model and a set of suspect operations that have logical relationships with a faulty execution. The size of this model is reduced in real time by the observation of controlled system. It helps to cut down the space of suspected elements in system. This is very important in using the $\mathrm{BN}$ for fault diagnosis in a complex manufacturing, due to it is impossible to learn all system variables and all production information for model identification. Besides, following with dynamic production environment, the $\mathrm{BN}$ need the time for its structure update, so that leads to additional cost and poor maintenance. Whereas, the Logical Diagnosis model executes in real time and adapts well with the changes of production environment.
In this paper, the suspect operations are considered as possible origins $\left\{O_{1}, \ldots O_{i}, \ldots O_{n}\right\}$ of detected fault. The reduced model describes the logical links between possible fault origins; thus, these logical links are considered as the causeconsequence relationships according to the failure propagation. The form of reduced model is a directed graph in which the nodes represent suspect operations, while the arcs represent the paths of failure propagation through product flows. The set of possible fault origins and the information of reduced model will be used for structural identification of $\mathrm{BN}$ model; and therefore the conditional probabilities are computed next. The main points of $\mathrm{BN}$ theory are presented in the next section.

\section{BAYESIAN NETWORK THEORY}

There are a number of studies that present presented the Bayesian network $(\mathrm{BN})$ theories such as [3], [4]. In literature, a $\mathrm{BN}$ is defined by:

- a directed acyclic graph $G, G=(V, E)$, where $V$ is the ensemble of nodes of $G$, and $E$ is the ensemble of edges of $G$.

- $\quad$ a finite probabilistic space $(\Omega, Z, P)$, with $\Omega$ is a nonempty space, $Z$ is a collection of subspace of $\Omega$, and $P$ is a probability measure on $Z$ with $P(\Omega)=1$.

- $\quad$ an ensemble of random variables associated to the nodes of $\mathrm{G}$ and defined on $(\Omega, Z, P)$, such as:

$$
P\left(V_{1}, V_{2}, \ldots, V_{n}\right)=\prod_{i=1}^{n} P\left(V_{i} \mid C\left(V_{i}\right)\right)
$$

where $C\left(V_{i}\right)$ is the ensemble of causes (parents) of $V_{i}$ in the graph $G$.

The Bayes rule is given by the equation (2):

$$
P(X \mid Y)=\frac{P(Y \mid X) \cdot P(X)}{P(Y)}
$$

- $\quad P(X \mid Y)$ is the conditional probability of $X$ given by $Y$.

- $P(Y \mid X)$ is the likelihood function of $Y$ given $X$.

- $\quad P(X)$ and $P(Y)$ are the prior probabilities of $X$ and $Y$.

When we need to extract the distribution over some subset of variables or a single variable, we need to marginalize or sum out the variables other than the variables of interest as explained in [19]. The marginalization rule for any sets of variable $\mathrm{X}$ and $\mathrm{Y}$ is given by :

$$
P(X)=\sum_{y} P(X, y) ; y \in Y
$$

The distribution over $\mathrm{X}$ can be obtained by summing out all the other variables from any joint distribution containing $X$. We can use the conditional probabilities instead of joint probabilities to compute the probabilities over $\mathrm{X}$ as shown in Equation (4):

$$
P(X)=\sum_{y} P(X \mid y) \cdot P(y) ; y \in Y
$$




\section{DIAGNOSIS MODEL}

\section{A. Model description}

The proposed model comprises of logical diagnosis model and BN model as shown in Fig.5. In this model, we use the results given by a logical diagnosis model introduced in Section 4 for the dynamically structural identification of a BN. After that, the probability values are computed by the BN model to support the decision-making for corrective maintenance.

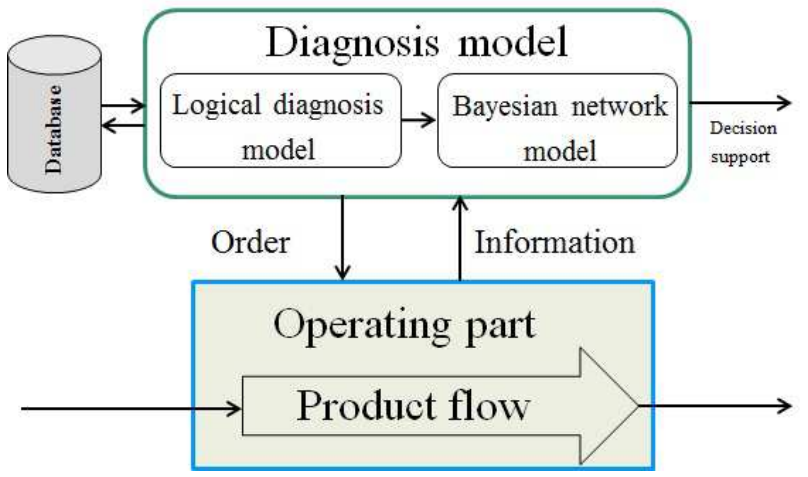

Fig. 5. Diagnosis model

The model for diagnosis is based on the control system and dynamic reconfiguration as proposed in [6], [11]. The reactive loop is characterized by collaboration of several supervision, monitoring and control (SM\&C) functions such as detection, diagnosis, prognosis, decision and automatic control [20]. The coordination level of control system has capacity to manage a set of Functional Chains and corresponding information. It demands and memorizes the information from the production database. It also provides the information for diagnosis.

Our methodology consists of: (i) searching possible root causes against a detected fault in the past evolution of the operating part in controlled system, (ii) computing the probability values that show the suspect levels against candidates. The process of diagnosis model execution is illustrated as following principles:

- This model is generated by the coordination level of a control system. This coordination level sends commands and receives reports in real time for and from all system components. This coordination level also provides the necessary information for the logical diagnosis.

- Once a failure is detected by a metrology, a set of possible origins and its correlations are defined by the logical diagnosis model.

- This set of possible origins and corresponding information are sent to BN model. Thus, a graphical structure of failure mode is determined to support the structural identification in learning step of BN model.

- After the establishment of graphical structure of BN model, the conditional probabilities associated with all nodes of network are computed based on historical information in the production database. All computed results are stored in the production database to support decision-making for corrective maintenance.

\section{B. The execution of diagnosis model}

When a fault is detected by a metrology, the diagnosis model manages its execution and will demand the diagnosis results from Logical Diagnosis model and the $\mathrm{BN}$ model. The Logical Diagnosis model provides a reduced model of the set of possible fault origins $\left\{O_{1}, \ldots O_{i}, \ldots O_{n}\right\}$ as presented in Section 4. This information is sent to $\mathrm{BN}$ through the coordination level, and it is used to construct the BN model. The conditional probabilities are computed based on the principal theories as presented in Section 5.

The BN model for diagnosis will be established and performed as follows:

- First, an algorithm as proposed below (Algorithm 1) is used for equivalent transition from a set of possible root causes and its directed graphical structure given by logical diagnosis model to a graphical structure of BN model. In Algorithm 1, we assume that a set of possible root causes has $\mathrm{n}$ members as $\left\{O_{1}, \ldots O_{i}, \ldots O_{n}\right\}$. Each member $O_{i}$ of the set obtained by logical diagnosis model is considered as one node in the BN. The other members $O_{i^{\prime}} ;\left(i^{\prime}=\right.$ $\left.1 \cdots n, i^{\prime} \neq i\right)$ are the parents of node $O_{i}$ if they are in front of the node $O_{i}$ and have directly logical relationship with $O_{i}$ following a product flow in directed graph.

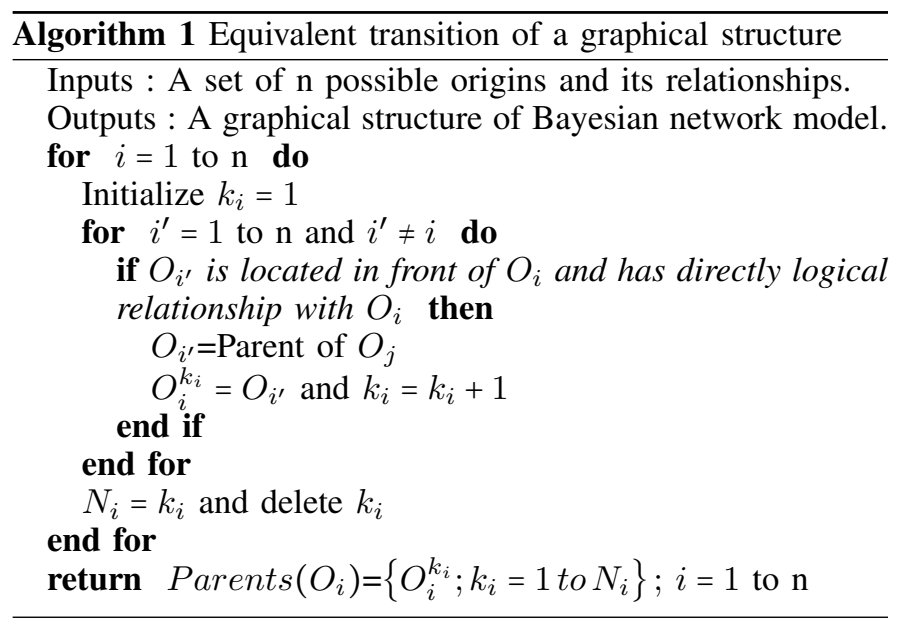

- $\quad$ Second, we obtain a graphical structure of BN model. Each node in this structure may be a parent of childnodes and may be a child of other parent-nodes. For instance, a set of parents a detected fault node is a finite number of mutually exclusive operation $\left\{O_{1}, \ldots O_{j}, \ldots O_{N}\right\}$. Each operation $O_{j}$ with $(j=$ $1 \cdots N)$ has a set of parents as $\left\{O_{j}^{1}, \ldots O_{j}^{k_{j}}, \ldots O_{j}^{N_{j}}\right\}$, with $k_{j}=1 \cdots N_{j}$. Therefore, each member $O_{j}^{k_{j}}$ has a set of parents as $\left\{O_{j}^{k_{j}, 1}, \ldots O_{j}^{k_{j}, l_{j}}, \ldots O_{j}^{k_{j}, L_{j}}\right\}$ with $l_{j}=1 \cdots L_{j}$. In this case, the $\mathrm{BN}$ model has a hierarchical structure as shown in Fig.6. 


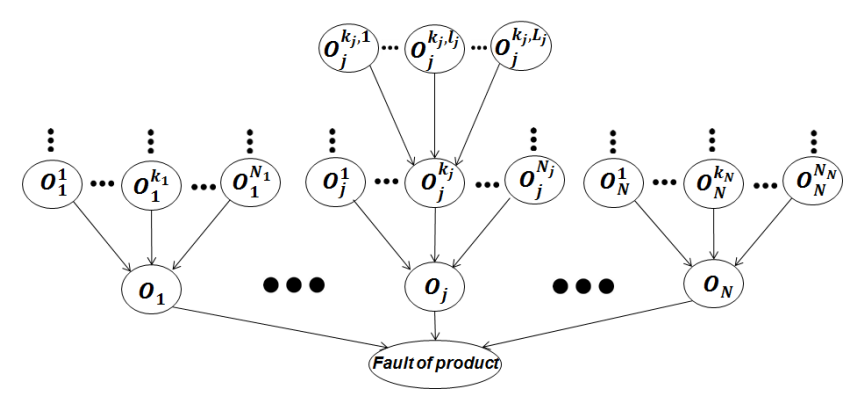

Fig. 6. Graphical structure of BN model

- Third, after a graphical structure is identified, the learning step of BN model will be performed to calculate the probabilities $P\left(O_{i} \mid\right.$ Product $)$ of each node $O_{i}$ following the product, and next the conditional probabilities between the child-nodes and its parents based on the historical information of production system from the database. We consider that each member of the set of fault origins has two states $\{1,0\}$. Thus, the conditional probabilities over each operation $O_{j}$ with $(j=1 \cdots N)$ and its parents $\left\{O_{j}^{1}, \ldots O_{j}^{k_{j}}, \ldots O_{j}^{N_{j}}\right\}$ are defined as a matrix $P\left(O_{j} \mid O_{j}^{1}, \ldots O_{j}^{k_{j}}, \ldots O_{j}^{N_{j}}\right)$ as shown in equation 5 :

$$
\left(\begin{array}{c}
p\left(O_{j}=1 \mid O_{j}^{1}=1, \ldots O_{j}^{k_{j}}=1, \ldots O_{j}^{N_{j}}=1\right) \\
\vdots \\
p\left(O_{j}=1 \mid O_{j}^{1}=0, \ldots O_{j}^{k_{j}}=0, \ldots O_{j}^{N_{j}}=0\right) \\
p\left(O_{j}=0 \mid O_{j}^{1}=1, \ldots O_{j}^{k_{j}}=1, \ldots O_{j}^{N_{j}}=1\right) \\
\vdots \\
p\left(O_{j}=0 \mid O_{j}^{1}=0, \ldots O_{j}^{k_{j}}=0, \ldots O_{j}^{N_{j}}=0\right)
\end{array}\right)
$$

- Finally, the model computes the conditional probabilities $P\left(O_{i} \mid\right.$ fault, Product $)$ over node $O_{i}$ with $(i=1 \cdots n)$ given by detected fault according to the Product as illustrated in the next equation:

$$
\begin{array}{r}
P\left(O_{i} \mid \text { fault, } \text { Product }\right)=\prod_{j=1}^{n} \sum_{O_{j}} P\left(O_{i}\right) . P\left(O_{j} \mid O_{i}\right) . \\
P\left(\text { fault } \mid O_{1}, \ldots, O_{j}, \ldots, O_{n}, \text { Product }\right)
\end{array}
$$

with $j=1 \cdots n, j \neq i$. They are used to support a decision of a maintenance operator.

The above sections show that a set of possible origins is dynamically determined when a fault is detected. And when the data is available, the corresponding conditional probabilities are calculated by diagnosis model. This set of possible origins and these probability values are sent and stored in the production database by the coordination level.

\section{Application capacity of proposed diagnosis model}

The advantages of proposed model are: first to locate a possible fault origins set in real time, second to reduce the space of this set by the evaluation of suspect levels, and finally less workload for structure identification of BN model. In the proposed model, the set of possible root causes is significantly reduced by the Logical Diagnosis model. Next, this is used to simplify a structural identification of BN model. Hence, the $\mathrm{BN}$ model receives a graphical structure with only elements at the possible origins of a detected fault. While other elements not related to the detected fault are removed. Consequently, it does not need to compute all probabilities of all elements in the production system.

In addition, the probabilistic values given by $\mathrm{BN}$ model help to continue reducing the set of possible origins through risk priority. This shows that we can save the recovery time of a production system. This also implies that the combination of a deterministic approach (logical diagnosis) and a probabilistic approach (Bayesian network) help us to locate more accurately and quickly equipment as the root cause of a detected fault. Consequently, this proposed model is feasible to apply for fault diagnosis in complex automated manufacturing systems with large production information.

The next section presents an example to explain the application of proposed diagnosis model in an automated production system.

\section{APPLICATION EXAMPLE}

To illustrate the basic principles of proposed method, we propose an example that consists of two cylinders as shown in Fig.7. The product is transported from position A to position $\mathrm{B}$ by the cylinder 1 and from position $\mathrm{C}$ to position $\mathrm{D}$ by the cylinder 2. A conveyor is used to transfer the product between positions B, C, E. Three sensors SA, SD, SE are installed in positions $\mathrm{A}, \mathrm{D}, \mathrm{E}$ to observe the position of a product. The goal of this system is to transport the products to position D. These monitoring sensors allow the verification of the values of product state variables. We consider that this production system is integrated with a diagnosis model that has a diagnosis mechanism as proposed in above section. The coordination level observes and qualifies the execution of all operations that are executed by the controlled system.

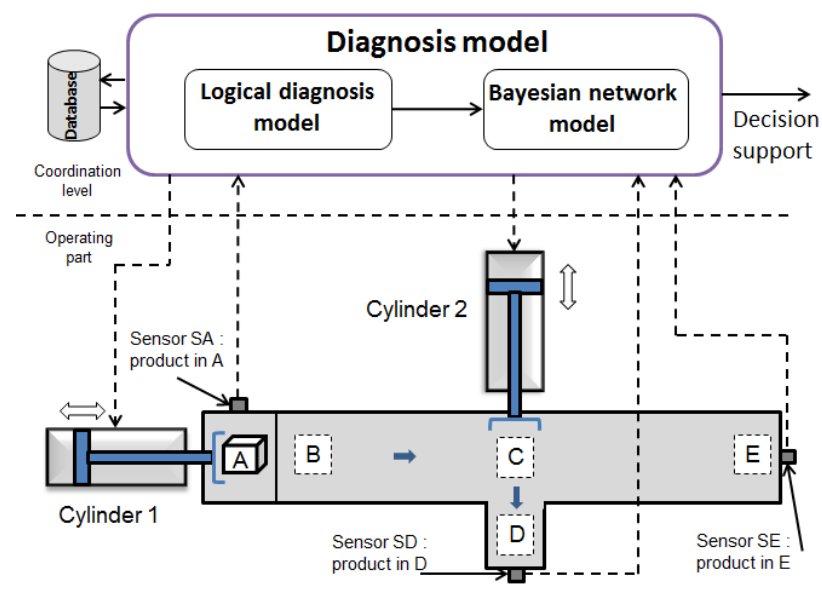

Fig. 7. Example to illustrate the application of diagnosis model

Following the operation to put product in position A, if the Functional Chain (FC) of sensor SA does not send a faulty execution report to the coordination level, it means that this FC has correlation between the product observation and information on production system behavior. Hence, the 


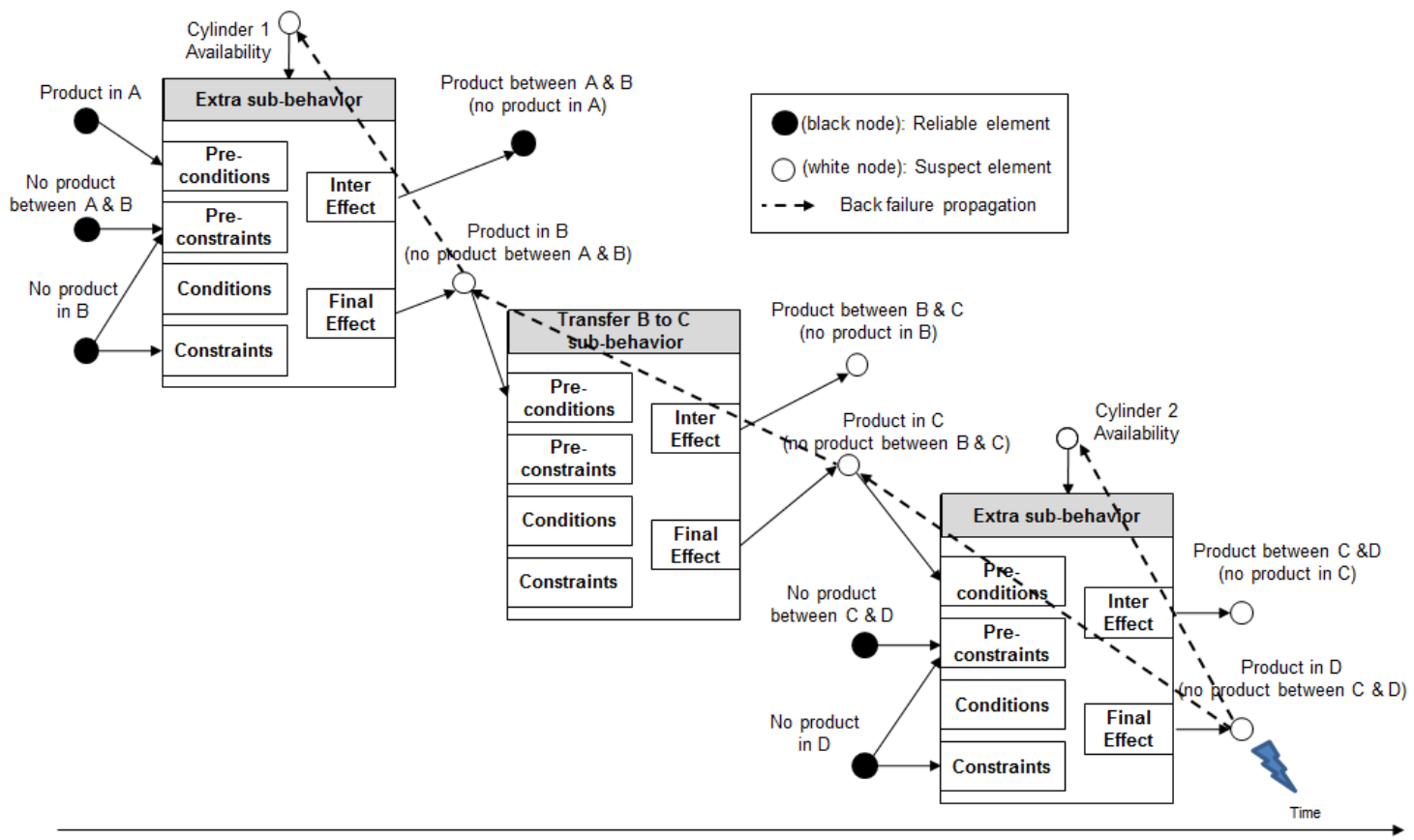

Fig. 8. Evolutions model following the product flows

corresponding (Pre-)conditions and (Pre-)constraints are also qualified as correct, then corresponding nodes (colored in black) are removed from the model. Finally, we obtain a reduced model that contains the suspect elements (the white nodes in Figure 8). When product is not detected at D, the FC of sensor SD send a faulty execution report to the coordination level. The mechanism based on a failure propagation approach is used to search for reduced model with the possible origins of this fault.

According to the faulty report of Product at position D, a set of possible origins consists of $\{$ Cylinder 1 , Product in B, Product in $C$, Cylinder 2$\}$ followed by the failure propagation. This set is transformed to a graphical structure of BN model by Algorithm 1 (presented in Section 4). This graphical structure and the conditional probabilities between the parent-nodes and child-notes are presented in Fig.9.

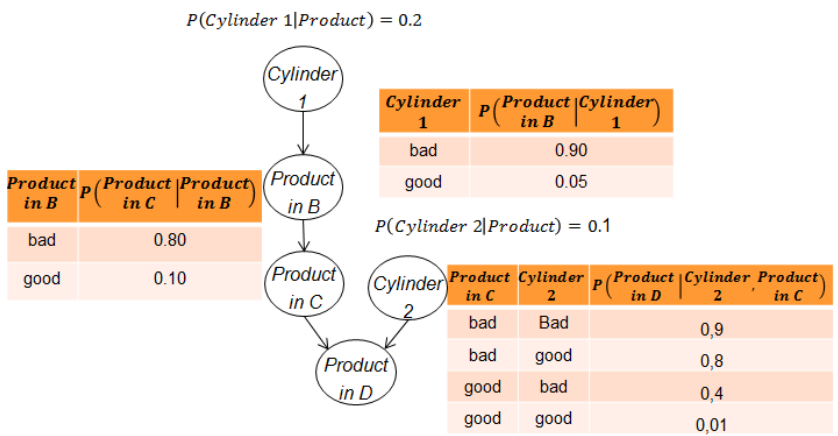

Fig. 9. The graphical structure and the conditional probabilities of its nodes

The Fig.9 illustrates the graphical structure for $\mathrm{BN}$ and the corresponding conditional probabilities. Where:

- $\quad P($ Cylinder $1 \mid$ Product $), P($ Cylinder 2|Product $)$ are the probabilities of Cylinder 1 and Cylinder 2 qualified as bad for a given product.

- $\quad$ P(Product in $B \mid$ Cylinder 1$)$ is the conditional probability of Product in $B$ given $C y l i n d e r ~ 1$.

- $\quad P($ Product in $C \mid$ Product in $B)$ is the conditional probability of Product in $C$ given Product in $B)$.

- $\quad$ (Product in $D \mid$ Cylinder 2, Product in $C)$ is the conditional probability of Product in $D$ given $C y$ linder 2) and Product in $C$.

The conditional probabilities of machines, which are given when product is in bad state, are shown as TABLE I.

TABLE I. THE PROBABILITY RESULTS

\begin{tabular}{l||c}
\hline $\boldsymbol{P}($ Cylinder $\mathbf{1} \mid$ Product in $\boldsymbol{D})$ & 0.0342 \\
\hline \hline $\boldsymbol{P}($ Product in $\boldsymbol{B} \mid$ Product in $\boldsymbol{D})$ & 0.0371 \\
\hline \hline $\boldsymbol{P}($ Product in $\boldsymbol{C} \mid$ Product in $\boldsymbol{D})$ & 0.2057 \\
\hline \hline $\boldsymbol{P}($ Cylinder $2 \mid$ Product in $\boldsymbol{D})$ & 0.0527 \\
\hline
\end{tabular}

The result shows that the possible origins of the fault detected on product by metrology may be one member of the set $\{$ Cylinder 1, Product in B, Product in C, Cylinder 2$\}$. Furthermore, the result shown in TABLE I concludes that the conditional probability $P($ Product in $C \mid$ Product in $D)$ of node Product in $\mathrm{C}$ corresponding to a given fault Product in $\mathrm{D}$ has maximum value. Consequently, the node Product in $\mathrm{C}$ must be first investigated by the personal maintenance.

This example also implies that if we do not use the BN, the set of possible origins have 4 members $\{$ Cylinder 1 , Product in $B$, Product in $C, C y l i n d e r 2\}$. Thus, we must investigate all of these 4 members as potential causes. However, if the $\mathrm{BN}$ model is used, the computed conditional probabilities help to make decision to investigate the member with highest suspect level. 
Moreover, if the diagnosis process is performed based on a BN model without the Logical Diagnosis model, all the nodes (black and white) in Fig.8 may be possible origins of detected fault at node Product in D. We must use a learning method to identify the graphical structure of the BN. The computation time and work-load become larger than the case of within Logical Diagnosis model.

In summary, the combination of two techniques allows making use of its advantages and avoiding inconveniences. The proposed model is dedicated to advance real time diagnosis and simplify the decision making for a maintenance strategy. It is appropriate to apply on the flexible manufacturing systems under dynamic production environment.

\section{CONCLUSION}

The proposed approach in this paper dynamically generates the structure of the $\mathrm{BN}$ and the associated probabilities. We used a Logical Diagnosis model to significantly reduce the search space for suspect equipment in the given production flow. This reduced set of possible origins as directed graph provides the cause-consequent relation to simplify the failure model identification in learning phase of BN. In addition, the associated probabilities are computed by the BN model evaluate the suspect level of each member in the set of possible fault origins. Our methodology has capacity to combine the advantages of both methods of Logical Diagnosis and BN.

In the context of complex manufacturing system that is characterized by multiple products, production lines, recipes and human factors. The elements of system often change under flexible production activities. The proposed model is appropriate in dynamically locating the root causes, in less time and less workload to compute the conditional probability values. The diagnosis results support decision-making for corrective maintenance activities.

This diagnosis objective is first based on the precisely and quickly locating the faults that come from equipment and products. Moreover, it must take into account the different fault sources as recipes and human factors; and furthermore their correlations. Consequently, our future work will improve on the fault diagnosis in a general manufacturing system with multiple sources of faults.

\section{REFERENCES}

[1] V. Venkatasubramanian, R. Rengaswamy, K. Yin, and S. N. Kavuri. A review of process fault detection and diagnosis: Part I: Quantitative model-based methods, Computers \& Chemical Engineering, vol. 27, no. 3, pp. 293-311, 2003.

[2] D. Ruiz, J. Canton, J. M. Nougues, A. Espuna, and L. Puigjaner, On-line fault diagnosis system support for reactive scheduling in multipurpose batch chemical plants, Computers \& Chemical Engineering, vol. 25, no. 4, pp. 829-837, 2001.

[3] P. Weber, G. Medina-Oliva, C. Simon and B. Iung. Overview on Bayesian networks applications for dependability, risk analysis and maintenance areas, Engineering Applications of Artificial Intelligence, volume 25, issue 4, pp. 671-682, 2012

[4] M.F. Bouaziz, E. Zamai,F. Duvivier and S. Hubac. Dependability of complex semiconductor systems: Learning Bayesian networks for decision support, 3rd International Workshop on Dependable Control of Discrete Systems (DCDS), pp. 7-12, 2011.

[5] O. Z. Maimon and L. Rokach (Eds.). Data mining and knowledge discovery handbook (Vol. 1), New York: Springer, 2005
[6] E. Deschamps and E. Zamai. Diagnosis for control system reconfiguration, In IFAC Management and Control of Production and Logistics, volume 4, no.1, pp. 377-382, 2007.

[7] S. Brown. Strategic manufacturing for competitive advantage: transforming operations from shop floor to strategy, Prentice Hall, 1996.

[8] E. J. Phillips. Manufacturing Plant Layout: Fundamentals and fine points of optimum facility design, Society of Manufacturing Engineers Dearborn, MI, 1997.

[9] A. Patterson Hine, G. Aaseng, G. Biswas, S. Narasimhan, and K. Pattipati. A review of diagnostic techniques for ISHM applications, 2005.

[10] A. Jones and A. Saleh. A multi-leve/multi-layer architecture for intelligent shopfloor control,International Journal of Computer Integrated Manufacturing, volume 3, issue 1, pp. 60-70, 1990.

[11] S. Henry, E. Zamai, and M. Jacomino.Controlled system model adapted for control law synthesis, 16th IFAC World Congress (IFAC05), Prague, Rpublic Tchque, 2005.

[12] J. White, D. Benavides, D.C. Schmidt, P. Trinidad, B. Dougherty and A. Ruiz-Cortes. Automated diagnosis of feature model configurations, Journal of Systems and Software, volume 83, issue 7, 1094-1107, 2010.

[13] N. Xiao, H.Z. Huang, Y. Li, L. He, and T. Jin. Multiple failure modes analysis and weighted risk priority number evaluation in FMEA, Eng. Fail. Anal, vol. 18, no. 4, pp. 1162-1170, Jun. 2011.

[14] S.Abdelwahed and G. Karsai. Notions of diagnosability for timed failure propagation graphs, In Autotestcon,IEEE, pp. 643-648, September, 2006.

[15] S. Strasser and J. Sheppard. Diagnostic alarm sequence maturation in timed failure propagation graphs, In AUTOTESTCON, IEEE, pp. 158$165,2011$.

[16] S. Khomfoi and L. M. Tolbert. Fault Diagnostic System for a Multilevel Inverter Using a Neural Network, IEEE Trans. Power Electron, vol. 22, pp. 1062-1069, 2007.

[17] Q.B. Duong, E. Zamai, K.Q. Tran-Dinh. Confidence estimation of feedback information for logicdiagnosis, Engineering Applications of Artificial Intelligence,volume 26, issue 3, pp. 1149-1161, 2013.

[18] R. M. Neal and G. E. Hinton. A view of the EM algorithm that justifies incremental, sparse, and other variants, In Learning in graphical models, Springer, pp. 355-368, 1998.

[19] C. E. Manfredotti. Modeling and inference with relational dynamic Bayesian networks, Advances in artificial intelligence, Springer, 2009.

[20] E. Zamai, A. Chaillet-Subias and M. Combacau. An architecture for control and monitoring of discrete events systems, Computers in Industry, volume 36, issues 1-2, pp. 95-100, 1998. 